\title{
Analysis of Cry in New Born Infants
}

\author{
Dhanashri U.S. Talauliker ${ }^{1}$, Nayana Shenvi \\ M.E (ECI), Electronics \& Telecommunication Department, Goa College of Engineering, Farmagudi- Goa, India ${ }^{1}$ \\ Electronics \& Telecommunication Department, Goa College of Engineering, Farmagudi- Goa, India ${ }^{2}$
}

\begin{abstract}
The acoustic analysis of infant cry is used to deduce information on the state of health of new-born babies as well as of children a few weeks old. Crying is the first tool of communication for an infant. These cries seem to be uniform, but there are a lot of differences between two infant's cries. Infant cry characteristics reflect the development and possibly the integrity of the central nervous system. The preterm infants and infants with neurological conditions have different cry characteristics like fundamental frequency, when compared to healthy full term infant. There are differences between full term and preterm infant in their neuro-physiological maturity and its impact on their speech development. Cry characteristics of New born infant, changes with increase in age. Acoustics analysis of infant cry signals can thus give an aid to clinical diagnosis and prevention of distress since it is easy to perform, cheap and completely non-invasive. Hence, this paper aims at pre-processing to eliminate silenced region of cry signal and estimating the fundamental frequency (pitch) using time domain and frequency domain analysis. Such parameters are of interest in exploring brain function at early stages of child development, for the timely diagnosis of neonatal disease and malformation.
\end{abstract}

Keywords: Fundamental frequency, Formants, Cry characteristics, Cry signal

\section{INTRODUCTION}

Cry is not just an infant behavior, but rather it is a part of a behavioral system in the human species that assures survival of the helpless neonate by eliciting others to meet basic needs. The cry signal results from coordination among several brain regions that control respiration and vocal cord vibration from which the cry sounds are produced. There is a relationship between acoustic characteristics of the cry and diagnoses related to neurological damage, Sudden Infant Death Syndrome, prematurity and substance exposure during pregnancy. Assessment of infant cry provides a window into the neurological and medical status of the infant. Assessment of infant cry is brief and noninvasive and requires recording equipment and a standardized stimulus to elicit a pain cry. It involves 30 seconds of crying from a single application of the stimulus. The recorded cry is submitted to an automated computer analysis system that digitizes the cry and either presents a digital spectrogram of the cry or calculates measures of cry characteristics. The most common interpretation of cry measures is based on deviations from typical cry characteristics. Infants with abnormal cries should be referred for a full neurological evaluation. Crying is a biological siren, alerting the care giving environment about the needs and wants of the infant and motivating the listener to respond. There are two key aspects of cry (1) the cry itself, which is innervated by the cranial nerves modulating the autonomic nervous system and signals emergency status, and (2) the salience of the cry to any potential caretakers in the environment, producing a visceral reaction that compels action.

\section{METHODS}

\section{A. "Cry": An Acoustic Signal}

Infant crying comprises the rhythmic alteration of cry sounds (utterances) and inspirations. Crying is part of the expiratory phase of respiration with sound or phonation produced by the larynx, which contains the vocal cords or folds and glottis (opening between vocal folds). The larynx has three functions: swallowing, breathing (glottis is fully open), and voice production (glottis is closed). When air is forced through adducted (closed) vocal cords, the increased air speed due to passage through a constricted tube, results in a drop in air pressure, causing the vocal cords to open and close rapidly (approx. 250 to $450 \mathrm{~Hz}$ or cycles per second in a normal healthy newborns). This vibration is the fundamental frequency (f0) and is heard as the pitch of the cry. In addition to f0, the lower vocal track produces sound characteristics such as loudness, and the rhythm of expiratory cry sounds and inspiration, which includes inhalation as well as breath holding. The upper vocal tract shapes the sound to produce resonant frequencies or formants (frequency bands above f0). The infant vocal tract is smaller than the adult vocal track.[2]

There are three identifiable cry modes of vocal fold vibration: basic cry or phonation or fundamental frequency (f0), high pitch cry or hyper-phonation (1000 to $2000 \mathrm{~Hz}$ ), and noisy or turbulent cry (dysphonation). The brainstem controls the contour and cross-sectional airway of the supraglottal system, which shapes formant frequencies. Only the first two formants are usually measured: The first formant (F1) occurs at approximately $1100 \mathrm{~Hz}$ and the second formant (F2) at approximately $3300 \mathrm{~Hz}$.

\section{B. Estimation of Fundamental Frequency}

Speech signal can be classified into voiced, unvoiced and silence regions. The periodic vibration of vocal folds is excitation for the production of voiced speech. The random like excitation is present for unvoiced speech. There is no excitation during silence region. Unvoiced 
sounds, or turbulences, are produced by forcing air through a constriction in the vocal tract, and voiced sounds are generated by forcing air through the glottis. The voiced regions look like a near periodic signal in the time domain representation.

The periodicity associated with such segments is defined is 'pitch period To' in the time domain and 'Pitch frequency or Fundamental Frequency 'fo' in the frequency domain. Pitch is an important attribute of voiced speech. It contains speaker specific information.

C. Method 1: Pitch estimation by Autocorrelation method (Time domain Analysis)

The information about pitch period 'To' is more pronounced in the autocorrelation sequence of voiced speech compared to the speech segment itself. The second largest peak is the autocorrelation sequence, represents 'To' and can be picked up easily by a simple peak picking algorithm. There is no prominent peak in the case of unvoiced speech. This is the fundamental distinction between voiced and unvoiced speech. Speech pitch is typically in the range $100-400 \mathrm{~Hz}$ and accordingly the pitch in the range $2.5-10 \mathrm{msec}$. Therefore for the estimation of pitch, the largest peak in the partial autocorrelation sequence starting from $2.5 \mathrm{msec}$ lag is found out and its distance with respect to zero lag is measured as pitch peak 'To'. Once To is known, then pitch can be computed as 1/To. The flowchart is as shown.

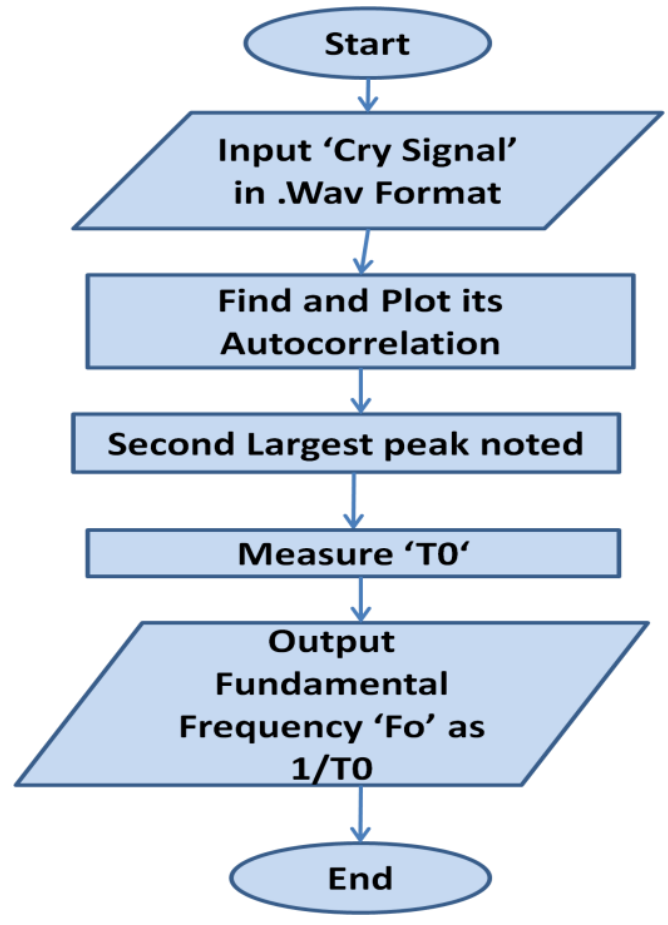

D. Method 2: Cepstrum Pitch Determination method (Frequency domain Analysis)

Main limitation of pitch estimation by the auto correlation of speech is that there may be peaks larger than the peak corresponding to the pitch period T0. As a result there may be picking of wrong peaks and hence wrong estimation of pitch. The approach to minimize such errors is to separate the vocal tract and excitation source related information in the speech signal and there use the source information for pitch estimation. The cepstral analysis of speech provides such an approach. The cepstrum of speech is defined as the inverse Fourier transform of the log magnitude spectrum. The cepstrum projects all the slowly varying components in log magnitude spectrum to the low frequency region and fast varying components to the high frequency regions. In the $\log$ magnitude spectrum, the slowly varying components represent the envelope corresponds to the vocal tract and the fast varying components to the excitation source. As a result the vocal tract and excitation source components get represented naturally in the spectrum of speech

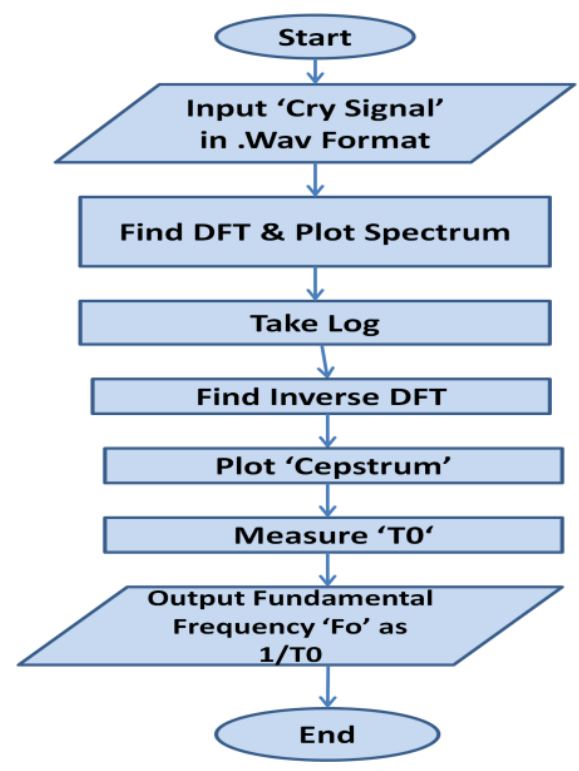

\section{RESULTS}

MATLAB 7.1 is used for coding. Codes are developed for representing cry signal in time \& frequency domain and estimating fundamental frequency in time \& Frequency domain. Also silenced region of cry signal has been removed for reducing the processing time. The results are as follows.

Removal of unvoiced (silenced region) segment from cry signal

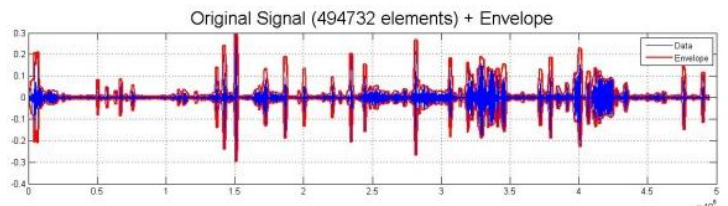

Edited Signal (124140 elements), shorter because data was removed

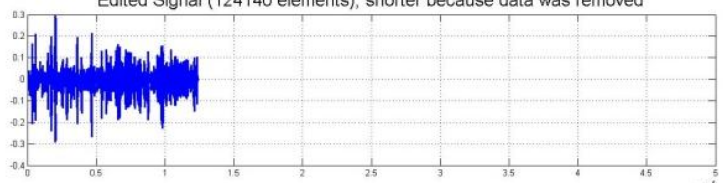


1) Representation of cry signal in time domain, frequency domain and its Spectrogram
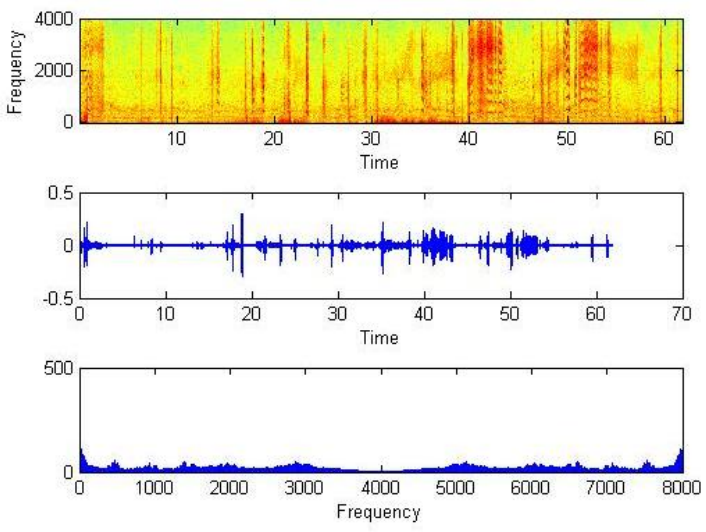

2) Estimation of Fundamental Frequency in time domain (using Autocorrelation)
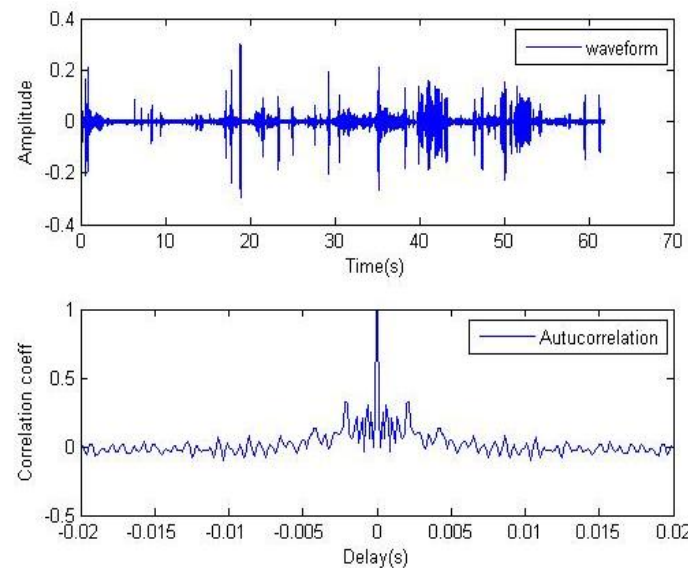

3) Estimation of Fundamental Frequency in frequency domain (Cepstrum analysis)
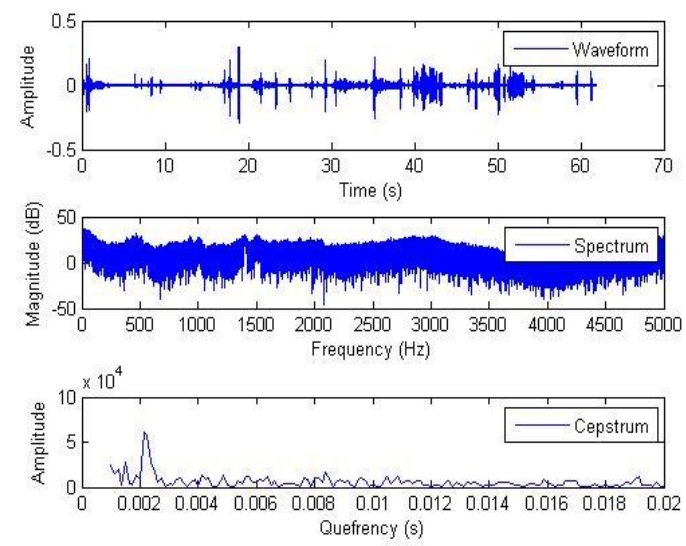

IV. CONCLUSION

The processing time of signal is reduced since silenced regions were removed. The Fundamental frequency found using two analyses are almost same and are in the range of normal infant.

fo $=444.444 \mathrm{HzHz}$ ( Using Autocorrelation method)

fo $=470.588 \mathrm{~Hz}$ ( Using Cepstrum Analysis)

\section{REFERENCES}

[1] Manfredi, I. Bocchi, S. Orlandi, L. Spaccatera, G.P. Donzelli," High resolution cry analysis in preterm newborn infants" Dept of E\&TC,Universita degli Studi di Firenze, Via S.Ma, Italy \& Dept of paediatrics, Children Hospital A.Meyer. Universita degli Studi di Firenze,Firenze, Italy,2009

[2] Linda L. LaGasse,A.Rebecca Neal and Barry M. Lester, "Assessment of infant cry: acoustic cry analysis and parental perception" Dept of pediatrics, Brown medical school, Rhode island, 2005

[3] Dror Lederman ,"Estimation of Infants' Cry Fundamental Frequency Using a Modified SIFT algorithm", 2010

[4] Yasmina Kheddache, Chakib Tadj "'Characterization of Pathologic Cries of Newborns Based on Fundamental Frequency Estimation", October 2013

[5] Raina P. Daga and Anagha M. Panditrao, "Acoustical Analysis of Pain Cries' in Neonates: Fundamental Frequency”, Special Issue of International Journal of Computer Applications on Electronics, Information and Communication Engineering - ICEICE No.3, Dec 2011

[6] Rami Cohen1, Yizhar Lavner, IEEE 27-th Convention of Electrical and Electronics Engineers in Israel, "Infant Cry Analysis and Detection", 2012

[7] Ada Fort, Claudia Manfredi, "Acoustic analysis of newborn infant cry signals", Medical Engineering \& Physics 20 (1998) 432-442

[8] G. Várallyay, Jr. Z. Benyó, A. Illényi, Z. Farkas, L. Kovács, "Acoustic analysis of the infant cry: classical and new methods", Proceedings of the 26th Annual International Conference of the IEEE EMBS San Francisco, USA, September, 2004

[9] Nemir Ahmed Al-Azzawi, "Automatic Recognition System of Infant Cry based on F-Transform", International Journal of ComputerApplications(0975-8887)Volume102-

No.12,September2014 